\title{
Mitochondrial disorders: aetiologies, models systems, and candidate therapies
}

\author{
G. Jane Farrar, Naomi Chadderton, Paul F. Kenna, and Sophia Millington-Ward
}

Smurfit Institute of Genetics, School of Genetics \& Microbiology, Trinity College Dublin, Dublin 2, Ireland

It has become evident that many human disorders are characterised by mitochondrial dysfunction either at a primary level, due to mutations in genes whose encoded products are involved in oxidative phosphorylation, or at a secondary level, due to the accumulation of mitochondrial DNA (mtDNA) mutations. This has prompted keen interest in the development of cell and animal models and in exploring innovative therapeutic strategies to modulate the mitochondrial deficiencies observed in these diseases. Key advances in these areas are outlined in this review, with a focus on Leber hereditary optic neuropathy (LHON). This exciting field is set to grow exponentially and yield many candidate therapies to treat this class of disease.

\section{Mitochondrial dysfunction in human disorders}

The mitochondrion, an intracellular organelle believed to have originated from an aerobic bacterium some 1.5 billion years ago [1], provides an essential supply of cellular energy in the form of ATP generated via oxidative phosphorylation (OXPHOS) with hundreds of mitochondria populating the cytoplasmic compartment [2] (Figure 1). Given the essential role of these organelles in cellular bioenergetics, it is not surprising that mitochondrial dysfunction is a hallmark of many heritable and acquired disorders. The advent of genetic linkage and next-generation sequencing (NGS) technologies has led to significant insights into the role of mitochondrial mutations in driving disease processes [3,4]. Mitochondrial disorders can be subdivided into three classes: (i) primary mitochondrial disorders caused by mutations in mitochondrial genes; (ii) disorders with mutations in nuclear genes involved in mitochondrial function; and (iii) secondary disorders that arise from the accumulation of mitochondrial damage over time frequently involving neurodegenerative pathologies [5-8]. Tissues such as retina, brain, and muscle, which have enormous energy requirements, are particularly vulnerable to variations in mitochondrial function. Approximately $50 \%$ of mitochondrial disorders have an ocular phenotype [9]. In addition to dominant optic atrophy (DOA) [10] and LHON (Box 1 [11]), mitochondrial dysfunction has been linked to multifactorial diseases, including

Corresponding author: Farrar, G.J. (gjfarrar@tcd.ie).

Keywords: mitochondrial disease; gene therapy; Leber hereditary optic neuropathy. age-related macular degeneration (AMD) [7,12] and diabetic retinopathies [12], as well as complex disorders involving multiple tissues, including the eye, such as neuropathy, ataxia and retinitis pigmentosa (NARP) and Kearns-Sayre syndrome (KSS) [13,14].

The significant mitochondrial dysfunction characteristic of many disorders has galvanised interest in the development of methodologies to simulate mitochondrial disorders in animal models and to deliver gene and small molecule-based drugs to the mitochondria. Studies in animal models will be essential in the development of therapies for these diverse and frequently devastating clinical conditions. Here, we review recent work highlighting the role of mitochondrial dysfunction in disease and the progress made towards understanding and treating these diseases, with a particular focus on LHON, a genetically wellcharacterised primary mitochondrial disorder.

Molecular characteristics of mitochondrial dysfunction Screens for pathogenic mtDNA mutations initially focused on genes encoding components of OXPHOS, such as those implicated in complex I deficiency disorders [15], together with nuclear genes whose products participate in OXPHOS. Additionally, pathogenic mutations have been found in genes whose products facilitate translation and assembly of OXPHOS complexes, in genes implicated in the fission and fusion of these dynamic organelles, and in mtDNA maintenance [16]. Mutations can operate through quantitative effects on mtDNA numbers or qualitative effects on mtDNA function. Mutations in the nuclear-encoded mitochondrial-specific DNA polymerase $\gamma(P O L G)$ involved in mtDNA replication and maintenance [17] have been implicated in diverse neurodegenerative conditions, including mitochondrial recessive ataxic syndrome (MIRAS) and late-onset chronic progressive ophthalmoplegia [18]. Factors that modulate mitochondrial RNA (mtRNA) metabolism by influencing mtRNA production, processing, stability, and polyadenylation have also been implicated. Mutations in mitochondrial amino acyl-tRNA synthetases (mt-aaRSs), key players in the translational machinery that link amino acids to their cognate mitochondrial tRNAs, are involved in several disorders [19]. Mutations in specific mitochondrial tRNAs have been reported [20,21]; for example, position 3243 in the mitochondrial tRNA ${ }^{\text {LEU(UUR) }}$ underlies $80 \%$ of mitochondrial encephalopathy lactic acidosis and stroke-like episodes (MELAS) [21]. 


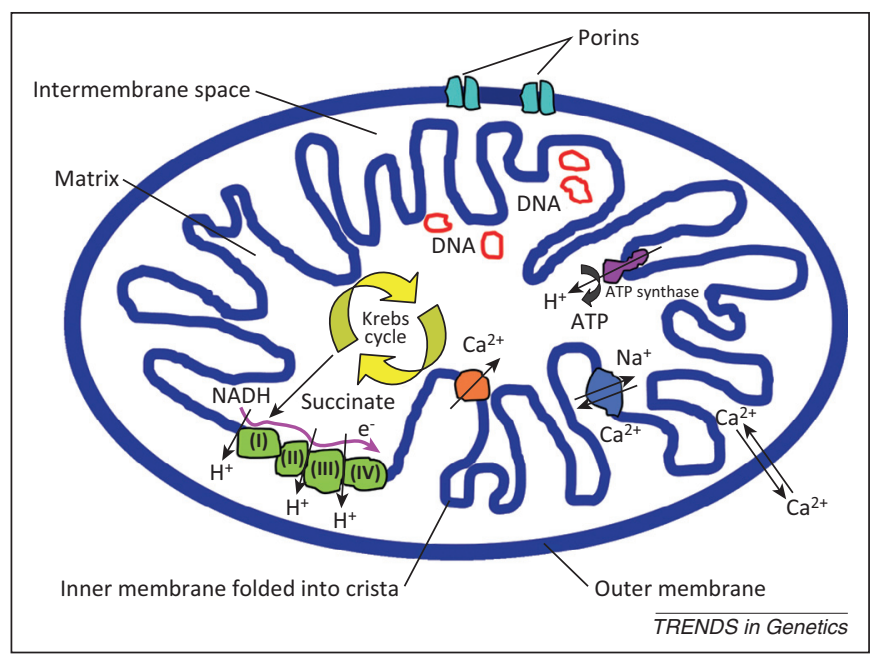

Figure 1. Diagrammatic representation of a mitochondrion. The inner compartment of the mitochondrion, the matrix, harbours among other components, the circular mitochondrial DNA (mtDNA). Within the matrix, acetyl co-enzyme A (CoA) is oxidised in the Krebs Cycle, resulting in the generation of $\mathrm{NADH}$, which is fed into the five complexes of the oxidative phosphorylation (OXPHOS) pathway located in the inner mitochondrial membrane (IMM), thereby creating energy in the form of ATP. The IMM and outer mitochondrial membrane also harbour, among others, sodiumcalcium exchangers and calcium uniporters, involved in maintaining calcium levels, and porins, which enable molecules to diffuse across the outer membrane. The IMM contains ATP synthase, which is an important energy-providing enzyme.

Many nuclear genes implicated in mitochondrial disease are inherited in an autosomal or X-linked recessive fashion, although dominant inheritance also occurs. By contrast, mutations in mtDNA are inherited maternally and involve hundreds or thousands of mitochondria within each cell, with multiple mtDNA genomes in every mitochondrion. Interindividual and intertissue variations in levels of heteroplasmy (ratio of mutant:wild type mtDNA) can greatly influence the severity of many mitochondrial diseases, including LHON [22]. Mitochondrial disorders are subject to many other factors that can influence the phenotype, including environmental modifiers; risk factors for LHON include smoking, solvent exposure, and antiretroviral medication [23-25]. Genetic modifiers may also impact on disease phenotype [26,27]; for example, an X-linked modifier locus for LHON has been reported [26]. Furthermore, certain mitochondrial backgrounds may modulate susceptibility to disease and this may be linked to variations in oxygen consumption, efficiency of electron transport, ATP generation, and reactive oxygen species (ROS) production [28]. Evidence suggests that for multifactorial disorders, such as AMD and Parkinson's disease (PD), specific mitochondrial haplogroups can aggravate or attenuate disease risk [29-33]. Evidence in support of mitochondrial haplogroups modulating disease is more compelling for disorders such as LHON and AMD than for PD and Huntington disease (HD), where equivocal findings have been reported.

Although great diversity exists in the nuclear and mtDNA mutations causative of mitochondrial diseases, at times there is a perturbation of common pathways, including ROS production, ATP synthesis, calcium homeostasis, regulation of the permeability transition pore (PTP), and mitochondrial biogenesis, suggesting the possibility of developing common therapies for these

\section{Box 1. Clinical and genetic characteristics of LHON}

LHON, a mitochondrially inherited optic neuropathy, predominantly affects young males (male:female ratio of 5:1) during the second and third decades of life, and involves degeneration of RGCs, loss of central vision, and atrophy of the optic nerve, often resulting in a rapid and devastating loss of vision. The disorder, which affects 1 in 14000 males, typically presents in a single eye as a painless loss of vision and is frequently followed by loss of vision in the second eye within months. One interesting aspect to this disorder is the observation that a small proportion of patients with LHON can show visual recovery with a diminution in symptoms over time for reasons that as yet remain elusive. Furthermore, other individuals of either gender can carry a known disease mutation, but remain asymptomatic. The incomplete penetrance in some individuals and the potential for visual recovery in others are aspects of LHON that are not well understood at a molecular level.

LHON is caused by mutations in genes encoding components of the mitochondrial respiratory $\mathrm{NADH}$-ubiquinone oxidoreductase complex (complex I). Seven of the 46 subunits of mammalian complex I are encoded by the mitochondrial genome (ND1-6 and ND4L). Mutations in three subunits, G3460A in ND1, G11778A in ND4 and T14484C in ND6, account for most LHON cases. LHON demonstrates many of the features of mitochondrial inheritance discussed in this review, with disease severity influenced by levels of heteroplasmy, mitochondrial haplogroups, nuclear modifier loci, and environmental factors. For example, the G11778A (ND4) and T14484C (ND6) LHON mutations on subgroups of a mitochondrial haplogroup J or the G3460A (ND1) mutation on the mitochondrial haplotype $\mathrm{K}$ background have been associated with increased risk of visual loss [29]. Differences between haplogroups may be partially due to mtDNA quantity, which influences OXPHOS capacity [30] and may account for gender differences in disease penetrance in LHON; there is a suggestion that oestrogens increase mtDNA levels, enhancing oxygen consumption and ATP generation [87].

conditions $[5,6]$. Although it is well established that somatic mtDNA mutations accumulate and mitochondrial function attenuates in ageing animals, the relative impact of ROS production, OXPHOS capacity, mtDNA replication errors, and mitochondrial biogenesis remains ill defined [34]. Indeed, there is some debate regarding the free radical theory of ageing, which purports that reduced OXPHOS capacity results in increased ROS levels, causing mtDNA mutation and thereby exacerbating reductions in OXPHOS capacity. For example, mutator mice (with a knock-in mtDNA polymerase gamma mutation [35]) (Table 1) have no or marginal increases in ROS production despite compromised OXPHOS capacity [34,36]. In exploring these complex interactions and determining the cellular consequences of mitochondrial mutations, cellular and animal models offer a powerful resource.

\section{Cellular models of mitochondrial disease}

Manipulation of the mitochondrial genome requires circumventing substantial barriers, including delivery to the thousands of mitochondrial genomes in a cell and transfer across the double membrane of the mitochondrion. Recapitulation of mitochondrial disorders in cell culture has largely relied on cytoplasmic hybrid cells or cybrids, in which the mitochondrial content of a host cell has been manipulated such that endogenous mtDNA has been depleted and repopulated with donor mtDNA from platelets or enucleated cells [37,38]. Cybrids have been used to characterise the disturbances in cellular bioenergetics 
Table 1. Animal models of mitochondrial disease ${ }^{a}$

\begin{tabular}{|c|c|c|c|}
\hline Model & Phenotype and/or model & Mode of generation & Refs \\
\hline \multicolumn{4}{|l|}{ Chemically induced models } \\
\hline MPTP induced (complex I inhibitor) & PD & Intraperitoneal injection & [89] \\
\hline NDUFS4 & $\begin{array}{l}\text { Ataxia and a failure to thrive; model for } \\
\text { Leigh syndrome }\end{array}$ & $\begin{array}{l}\text { Homozygous conditional knockout; } \\
\text { point mutation; transgenic }\end{array}$ & {$[47,90]$} \\
\hline PINK1 knockout & Impaired neural activity; model for PD & $\begin{array}{l}\text { G309D point mutation with near loss of } \\
\text { expression }\end{array}$ & [91] \\
\hline \multicolumn{4}{|c|}{ Allotopic expression of mitochondrial genes } \\
\hline ND4 R340H & LHON & $\begin{array}{l}\text { Intravitreal injection of AAV or plasmid } \\
\text { DNA followed by electroporation }\end{array}$ & {$[53,54]$} \\
\hline ATP6 L156R & NARP and MILS & $\begin{array}{l}\text { Mitochondrial gene expressed in the } \\
\text { nucleus; transgenic }\end{array}$ & [52] \\
\hline $\begin{array}{l}\text { Deletor mouse: mutation in replicative } \\
\text { helicase Twinkle }\end{array}$ & $\begin{array}{l}\text { Multiple mtDNA deletions late-onset } \\
\text { progressive respiratory-chain deficiency in } \\
\text { skeletal, muscle, and certain neuronal } \\
\text { populations; model for adult-onset } \\
\text { myopathy and neurodegeneration }\end{array}$ & $\begin{array}{l}\text { cDNA transgene with dominant mutation } \\
\text { in a normally nuclear encoded replicative } \\
\text { helicase Twinkle gene }\end{array}$ & {$[96,97]$} \\
\hline ANT1 knockout mouse & $\begin{array}{l}\text { Exercise intolerance, increase in } \\
\text { mitochondrial proliferation in skeletal } \\
\text { muscle, enlarged heart with multiple } \\
\text { mtDNA rearrangements; model for adult- } \\
\text { onset myopathy and cardiomyopathy } \\
\text { resulting from mtDNA deletions }\end{array}$ & $\begin{array}{l}\text { Homozygous targeted disruption of } \\
\text { nuclear ANT1 gene exons } 1-3\end{array}$ & [98] \\
\hline $\begin{array}{l}\text { Mice expressing a G14600A mutation } \\
\text { in ND6 gene }\end{array}$ & Simulated aspects of LHON & Use of ES cells and cybrids & [57] \\
\hline
\end{tabular}

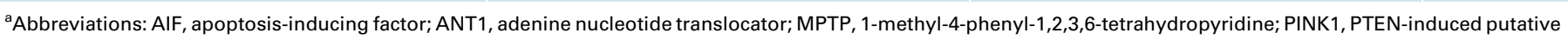
kinase 1.

associated with specific mitochondrial mutations. Examples include a partial tandem duplication of tRNA ${ }^{\mathrm{PHE}}$ in late-onset mitochondrial myopathy [39], causative of reduced oxygen consumption rates in cybrids, and the A3243G transition in the tRNA ${ }^{\mathrm{LEU}(U \mathrm{UU})}$ gene, which results in defects in respiratory chain assembly and activity, causative of MELAS, deafness, and cardiomyopathy [40]. The approach has also been adopted to evaluate the relative contribution of mitochondrial and nuclear genetic backgrounds in driving disease risk. Although cybrids represent valuable cellular models for experimentation, they do not recapitulate many of the multivalent features of disease in whole organisms. Moreover, studies typically have been limited to analyses using single cybrid cell lines, at times using cell types not directly relevant to the disease pathology, thereby mirroring only some aspects of disorders that frequently involve multiple cell types.
Cybrid and stem cell technologies have been combined to explore mitochondrial dysfunction in neurons differentiated from mouse embryonic stem (ES) cell cybrids carrying complex I and complex IV mutations, thereby generating cell types directly relevant to a condition with mitochondrial mutations [41]. Recently, depletion of endogenous mtDNA in human pluripotent stem cell-derived neural progenitors (hNPs) using dideoxycytidine and replacement with mutant mtDNA carrying the G11778A NADH dehydrogenase 4 (ND4) LHON mutation, together with delivery of donor mtDNA to hNP cybrids, was reported [42].

Other cell types have been utilised to generate cellular models of mitochondrial disease, including primary cells such as fibroblasts from skin biopsies $[43,44]$ or induced pluripotent stem (iPS) cells, thereby recapitulating mitochondrial disorders in patient-derived cells [45]. Although there are many applications for iPS cells, which are stem 
cells that have undergone exogenously directed reprogramming from nonpluripotent cells and that can subsequently be differentiated into cell types relevant to particular disease pathologies, limitations with the technology are evident. Significant variance has been observed between the behaviour of individual iPS clones and subclones after reprogramming, genetic and epigenetic differences between iPS-derived clones compared with endogenous cell types have been found, and, as with any cell model, the multifaceted nature of disease cannot be fully simulated. Various disorders addressed using primary cells and iPS methodologies include HD, PD, and Alzheimer's disease $(\mathrm{AD})$, all of which involve significant mitochondrial dysfunction in cells [46]. Despite these drawbacks, cybrids and iPS technologies have revolutionised the generation of cell models of disease, providing insights into disease pathogenesis and offering in vitro systems to screen candidate therapies [47]. Advances in methodologies to differentiate stem cells into homogeneous populations of specific cell lineages will improve the resolution of cell models and, therefore, their future utility to expedite elucidation of disease processes and to evaluate therapies. However, animal models of mitochondrial disease will remain invaluable for elucidating disease mechanisms and essential for preclinical evaluation of therapies.

\section{Animal models of mitochondrial disease}

Methodologies adopted to generate animal models include nuclear transgenesis of genes involved in OXPHOS and mtDNA maintenance, allotopic expression of mitochondrial genes, chemically induced models, and transmitochondrial approaches (Table 1) [47]. For disorders where the pathogenesis originates from mutations in nuclear genes whose encoded products participate in OXPHOS, standard methodologies for nuclear transgenesis can be used. A recent example is a transgenic model of Leigh syndrome, which is caused by mutations in the gene encoding NADH dehydrogenase (ubiquinone) iron-sulfur protein 4 (NDUFS4), a nuclear-encoded complex I subunit [48]. Transgenic mice with a conditional knockout of the NDUFS4 gene presented with a fatal progressive encephalopathy similar to human patients, regardless of whether the knockout was global or exclusive to the central nervous system (CNS), suggesting that NDUFS4 function is of particular relevance to the CNS. Although generating animal models mutant for nuclear-encoded components of OXPHOS is feasible, few models exist thus far $[47,49,50]$ (Table 1). Given the conserved nature of many OXPHOS components, in many cases conditional models will be required to overcome embryonic lethality associated with global mutants. Nuclear transgenesis has been used to generate mice with elevated rates of mitochondrial mutation due to mutations in the polymerase gamma A ( PolgA) gene $[35,50]$ encoding the nuclear subunit of mtDNA polymerase and involved in proofreading activity in mtDNA. Mutator mice simulate mtDNA genotypes and phenotypes seen in aged animals [35,50]. Deletor mice with higher rates of mtDNA mutation have been generated with mutations in Twinkle, which was recently shown to be the only helicase involved in mtDNA replication in mammalian mitochondria [51].
Modelling mtDNA mutations in animals presents greater challenges than does nuclear transgenesis, impeded by the multitude of mitochondrial genomes in a cell, the double membranes of the mitochondria, and selective pressures against mtDNAs that compromise OXPHOS. A method to circumvent such barriers has been allotopic expression in the nuclear compartment of a mitochondrial gene engineered with mitochondrial targeting sequences (MTS) facilitating protein import to mitochondria (Table 1). Such an approach has been applied in the generation of transgenic mice expressing wild type or L156R mutant forms of the mitochondrial ATP synthase 6 (ATP6) gene implicated in neuropathy, ataxia, and retinitis pigmentosa (NARP) and maternally inherited Leigh syndrome (MILS) [52]. With regard to LHON, intravitreal injection of plasmid DNA followed by electroporation [53] or an adenoassociated virus (AAV) [54] expressing a human ND4 complex I R340H mutant recapitulated features of human LHON, including retinal ganglion cell (RGC) and optic nerve degeneration; both studies used allotopic expression of ND4 but differing modes of delivery.

An alternative approach for the production of animal models has involved manipulation of mtDNA in ES cells. For example, xenomitochondrial mice with divergent nuclear and mtDNA backgrounds have been generated by injecting cybrid 129S6 ES cells containing Mus terricolor mtDNA into Mus musculus domesticus (C57BL) blastocysts. Backcrossing to C57BL mice for ten generations produced mice with a homogeneous nuclear background and divergent nuclear and mtDNA genomes [55]. As with animal models of nuclear disorders, simulating mitochondrial disorders in animals does not always result in a disease phenotype. Although preliminary studies with cybrid cells demonstrated mitochondrial dysfunction, xenomitochondrial mice exhibited no phenotype, suggesting compensatory mechanisms at play [55]. Although the process of mitochondrial depletion can result in cellular damage, transmitochondrial mice have been generated by fusion of cytoplasts containing mutant mtDNA with mitochondria-depleted mouse ES cells or by electrofusion of cytoplasts into mouse zygotes. These so-called 'mito-mice' carrying a mtDNA deletion encompassing 6tRNA and seven structural genes, a mutation in the genes encoding cytochrome oxidase I $(C O I)$ or $\mathrm{NADH}$ dehydrogenase 4 (ND6) have been generated [56]. Recently, using a homoplasmic G14600A ND6 mutant cell line, cybrids from this cell line were fused with ES cells depleted of mtDNA to generate a mouse with a mitochondrial mutation in ND6 [57] that recapitulates many features of LHON. The model suggests that LHON is due to oxidative stress rather than to ATP deficiency, because in this model, no reduction in ATP production was evident. Improved methods for mitochondrial transgenesis will aid animal model generation. Targeting of genetic modifications to specific cell lineages greatly increased the resolution of nuclear transgenic technologies and, likewise, tissue-specific manipulation of mtDNA would enhance the variety of models available for mitochondrial disorders. Development of potent methods for DNA delivery to mitochondria will find broad utility in the future generation of transgenic animals and in exploring novel therapies for mitochondrial disorders. 


\section{Delivering molecules to mitochondria}

Although an array of methods has been explored to achieve delivery of nucleotides and peptides to mitochondria [58-61], few data exist on the relative efficiencies of methodologies, levels of delivery to cytoplasm versus subcellular targets, proportions of mitochondria targeted, and longevity of delivered molecules. Nevertheless, delivery technologies under development are detailed below and provide an overview of the various approaches being considered. Biolistic methods enable DNA transfer to mitochondria using heavy particles coated with DNA; however, successful delivery has been limited to simple organisms, such as the yeast Saccharomyces cerevisiae [60]. Other strategies for delivery use nanocarriers, such as MITO-Porter, a liposome-based vector used for delivery to the mitochondrial matrix, in a process involving membrane fusion [62]; inclusion of a mitochondrial targeting signal (MTS) peptide with MITO-Porter enhanced mitochondrial delivery [63]. Liposome-like carriers derived from dequalinium (DQAsomes) have been used to deliver a minimitochondrial genome with a GFP gene into the mitochondrial compartment of macrophage cells using GFP fluorescence to monitor delivery [64]. The lipophilic cation triphenylphosphonium (TTP) has been used extensively to deliver nucleotide, peptide, and chemical molecules facilitated by the large mitochondrial plasma membrane potential (negative inside mitochondria), driving accumulation of this positively charged carrier within mitochondria. The approach was used to deliver the antioxidant MitoQ in animal models, prompting its evaluation in clinical studies $[65,66]$. Peptides, including mitochondrial-penetrating and Szeto-Schiller peptides, have been tested for their ability to direct delivery to mitochondria [6]. Indeed, water-soluble Szeto-Schiller peptides, which target the inner mitochondrial membrane (IMM), have antioxidant properties themselves, and are in preclinical and clinical development for several indications [67]. The recombinant human mitochondrial transcription factor A protein (TFAM), which itself stimulates mitochondrial biogenesis, has been used to deliver mtDNA to mitochondria, a strategy termed 'protofection'. Wild type mtDNA was delivered to near homoplasmic mutant mtDNA cybrid lines generated using platelets from patients with LHON or PD and primary fibroblasts from a patient with Leigh syndrome [44,68], resulting in significant improvements in mitochondrial respiration, biogenesis, and transcription in treated cells.

An alternative strategy to compensate for mitochondrial dysfunction involves delivery of polycistronic RNAs (pcRNAs) encoding parts of the mitochondrial genome [69]. General use of modified mRNAs is being considered as an alternative to delivery of double-stranded DNA to minimise immunostimulatory effects [70], a potential advantage of mitochondrial pcRNA delivery. A protozoanderived mitochondrial tRNA carrier complex has been used to facilitate mitochondrial delivery of pcRNAs in vivo in old rodents to increase the respiratory capacity of skeletal muscles, which typically decreases with age [69]. Furthermore, recent insights into the mammalian mtRNA transcriptome have demonstrated the presence of greater numbers of nuclear-encoded RNAs than previously envisaged, including tRNAs, miRNAs, and rRNA [71], revealing RNA import pathways for RNA delivery to mitochondria. For example, a 20-nucleotide stem-loop sequence from the RNA component of the RNAse P enzyme (H1) imported into mitochondria has been used to facilitate transfer of nuclear RNAs, both tRNAs and mRNAs, to mitochondria, an important observation in the field [72]. Knowledge gained from transcriptomics will expedite the development of additional methods for targeting nucleotide delivery to mitochondria, methodologies that will be applied in the future exploration of therapies for mitochondrial disorders. The exploitation of endogenous import mechanisms will likely provide strategies that target sufficient proportions of intracellular mitochondria to elicit readily quantifiable and potentially beneficial effects.

Table 2. Candidate gene therapies for mitochondrial disorders ${ }^{a}$

\begin{tabular}{|c|c|c|c|c|}
\hline Model and disease & Strategy & Therapeutic gene & Delivery & Refs \\
\hline $\begin{array}{l}\text { Mouse models and patients } \\
\text { with OTC deficiency }\end{array}$ & Gene replacement & OTC & AAV2/8 & {$[73,74,103]$} \\
\hline $\begin{array}{l}\text { Macaque monkey model for } \\
\text { PD induced by mitochondrial } \\
\text { complex I inhibitor MTPT }\end{array}$ & Gene replacement & $\begin{array}{l}\text { Tri-cistronic lentiviral } \\
\text { vector encoding tyrosine } \\
\text { hydroxylase, aromatic } \\
\text { L-amino acid decarboxylase } \\
\text { and guanosine } 5^{\prime} \text {-triphosphate } \\
\text { cyclohydrolase }\end{array}$ & Lentivirus, injection into striatum & [104] \\
\hline $\begin{array}{l}\text { Murine model for ethylmalonic } \\
\text { encephalopathy }\end{array}$ & $\begin{array}{l}\text { Gene replacement } \\
\text { to liver }\end{array}$ & ETHE1 & $\begin{array}{l}\text { AAV2/8-mediated gene transfer to } \\
\text { liver }\end{array}$ & [75] \\
\hline $\begin{array}{l}\text { Harlequin mice with complex } \\
\text { I deficiency-related } \\
\text { neurodegeneration }\end{array}$ & Gene replacement & AlF1 & $\begin{array}{l}\text { AAV2/2 intravitreal injection } \\
\text { followed by electroporation }\end{array}$ & [106] \\
\hline Two murine models for LHON & $\begin{array}{l}\text { Allotopic gene } \\
\text { replacement }\end{array}$ & ND4 & $\begin{array}{l}\text { AAV2/2 and electroporation in } \\
\text { vitreous }\end{array}$ & {$[53,54]$} \\
\hline Murine model for PD & Gene replacement & PINK1 & Ex vivo transfection & [110] \\
\hline
\end{tabular}

${ }^{a}$ Abbreviations: AIF1, apoptosis-inducing factor; MTPT, 1-methyl-4-phenyl-1,2,3,6-tetrahydropyridine; NDI1, NADH dehydrogenase subunit 1; OTC, ornithine transcarbamylase; PINK1, PTEN-induced putative kinase 1. 


\section{Candidate gene therapies for mitochondrial disorders} Nucleotide delivery methodologies, together with methods to optimise protein import into mitochondria, are being used to explore candidate therapies for mitochondrial deficiency disorders (Table 2). Most of the mitochondrial proteome (comprising approximately 1500 polypeptides) originates from proteins expressed from nuclear genes with signals (MTS) to facilitate import into mitochondria. This feature has been leveraged successfully in gene therapies for mitochondrial disorders and was first demonstrated decades ago in studies of ornithine transcarbamylase (OTC) deficiency, a urea cycle disorder. By using

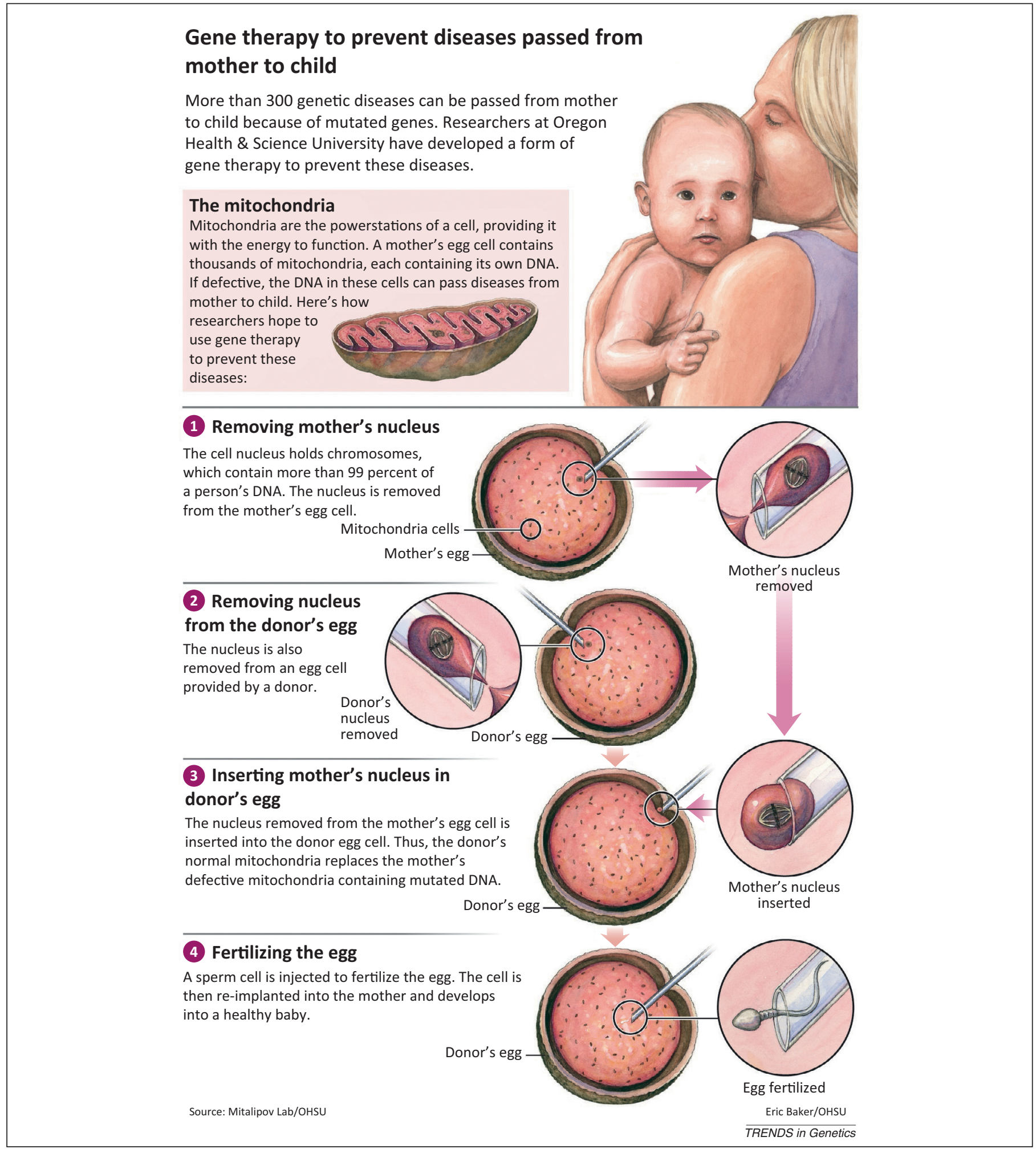

Figure 2. Diagrammatic representation of germline gene therapy for human mitochondrial disorders. There is growing interest in developing methodologies to circumvent the inheritance of mitochondrial disorders in the offspring of an affected parent by using a strategy involving the use of genetic material from three individuals: the mother, the father, and a donor female. In essence, the approach involves using an enucleated egg from a donor female into which the nucleus from the mother's egg is inserted, followed by fertilisation by the father's sperm of the 'hybrid' egg, whose mtDNA material originates from the donor female and nuclear material from the mother. Significant strides have been made recently towards this objective [83-86]. Reproduced with kind permission from Shoukhrat Mitalipov, Oregon Health \& Science University. 
the endogenous MTS, a nuclear-encoded wild type gene carried by adenovirus was delivered to mitochondria in an OTC-deficient mouse, resulting in correction of the defect [73]; recently, an AAV, the AAV2/8 serotype, has also been used for OTC gene therapy in rodents [74]. More recently, a similar approach exploiting mitochondrial import and an AAV2/8 vector was used in liver to treat mice with ethylmalonic encephalopathy, a fatal recessive disorder caused by mutations in the ethylmalonic encephalopathy 1 (ETHE1) nuclear gene encoding a mitochondrial sulfur dioxygenase involved in detoxifying hydrogen sulfide [75].

Allotopic expression of modified mitochondrial genes to facilitate nuclear expression and subsequent protein import into mitochondria overcomes the barrier of mitochondrial polyploidy and, hence, is also being used for therapeutic development. As with any gene therapy, the efficacy and longevity of the treatment will in part depend on the vector used for gene delivery. For example, DNA electroporation or AAV-mediated delivery into retinal ganglion cells of a wild type ND4 complex I subunit incorporating a MTS prevented RGC loss and optic nerve degeneration in mice expressing the ND4 R340H mutant [53,76], providing support for progression of ND4-based therapeutics for LHON towards clinical trial. In a recent study, inclusion of an MTS from the gene encoding mitochondrial cytochrome oxidase subunit 8 into the viral protein 2 (VP2) region of the AAV2 capsid directed delivery of the AAV cargo, the ND4 gene, to the mitochondrial compartment; however, the mode of action of this targeted delivery remains elusive [76].

An alternative gene therapy being considered for LHON utilises a trans-kingdom strategy using a single subunit nuclear-encoded complex I equivalent (the NDI1 gene) from yeast, the advantage being that it circumvents both the genetic heterogeneity underlying complex I-related LHON and the barrier of delivery to mitochondria. The NDI1 gene delivered using an AAV5 vector when stereotactically injected into the superior colliculus of the brain provided benefit in a rat model of LHON [77]. Recently, intravitreally injected AAV2/5 expressing NDI1 has been shown to provide significant benefit in a murine model of LHON [78], representing an important step forward because intravitreal viral administration is more translatable to the clinic.

Another avenue for treating mitochondrial disorders makes use of the observation that phenotypic expression of a mitochondrial disease can be influenced by levels of heteroplasmy [31]; hence, modulation of mutant:wild type mtDNA ratios may ameliorate disease symptoms. Ratio modulation can be achieved by targeted destruction of the mutant mtDNA [79], metabolically induced heteroplasmic shifting [40] (as observed in mutant cybrids grown in a low glucose regime), or delivery of wild type mtDNA genomes [42]. Systemic administration in neonatal mice carrying two neutral mtDNA variants of an AAV9 vector with the ApaLI restriction endonuclease engineered with a MTS to cleave one of the mtDNA variants resulted in heteroplasmic shifting in all striated muscles evaluated and demonstrated the utility of the approach to alter ratios of mtDNA variants [79]. Most recently, using what is termed an 'antigenomic' approach (which relies on complementary sequences), heteroplasmic shifting was achieved in cybrids by targeting oligoribonucleotides complementary to the boundaries of a large mtDNA deletion implicated in KSS, thereby preferentially preventing replication of the mutant mtDNA and resulting in a shift in wild type:mutant mtDNA ratios [14]. For some mitochondrial disorders, including LHON, mtDNA mutations are often homoplasmic, making such an approach inappropriate. As outlined above, delivery of wild type mtDNA also represents a rational therapeutic strategy being pursued [44] and may be used to modulate levels of heteroplasmy.

Various neurotrophic factors and antioxidants are being explored as therapies for neurodegenerative disorders, including LHON [80]. EPI-743, a small molecule whose mode of action may involve regulation of metabolic control, antioxidants effects, and or modulation of programmed cell death, is also being evaluated for LHON [81]. Idebenone, a coenzyme Q10 analogue (Santhera), is in a Phase III trial for LHON [82]. Although the beneficial effects thus far may be modest, to patients with LHON and the resulting devastating vision loss, they are welcome.

Unlike nuclear disorders, germline gene therapy is being considered for mitochondrial disorders and, indeed, significant strides have been made recently, with reports indicating that successful transfer of the nuclear genetic material from human oocytes carrying mutant mtDNA to enucleated recipient oocytes containing wild type mitochondrial genomes has been achieved $[83,84]$ (Figure 2). Of note, in previous studies, genome exchange between oocytes of nonhuman primates resulted in production of healthy offspring [85] and pronuclear transfer between fertilised human zygotes had been achieved [86]. This approach, once further developed, may allow females carrying mtDNA mutations to produce healthy offspring. Mitochondrial dysfunction is also involved in many sporadic cases of disease and tends to be characterised by an accumulation of mutant mtDNA throughout the lifetime of the individual. For such diseases, somatic gene therapy strategies rather than germline strategies will remain the focus.

\section{Concluding remarks}

Perturbations in mitochondrial function play a role in a wide array of diseases, providing enormous impetus to the scientific community to develop methods to treat or circumvent these conditions. Efficient delivery of nucleotide and peptide-based drugs to mitochondria represents a key objective, but is not trivial given the double-membrane structure of this organelle and the thousands of mitochondrial genomes resident in each cell. Many endogenous mitochondrial import pathways are being harnessed to aid drug delivery, and there have been some early successes with these approaches, although much remains to be done. Further exploration of the mitochondrial transcriptome and proteome should uncover additional pathways for import and expedite the future development of efficient delivery strategies. Although the rationale for treatment of symptomatic patients is obvious, the prophylactic use of future therapies in pre-symptomatic patients will need to be explored in the context of each emerging therapy and associated risks and benefits. Unlike gene 
therapies for disorders with a nuclear origin that thus far have been limited to somatic approaches, both somatic and germline strategies are being considered for mitochondrial diseases, as detailed above. Although further advances will be required in all of these areas, the stage is now set for an explosion in the development and evaluation of novel therapies targeting the mitochondrial deficiencies characteristic of so many human disorders.

\section{Acknowledgments}

Our work has been supported by Science Foundation Ireland, the Health Research Board of Ireland, Fighting Blindness Ireland, Foundation Fighting Blindness USA (NNRI), the Wellcome Trust, the European framework programme EviGenoRet, Enterprise Ireland and the Irish Research Council for Science, Engineering and Technology.

\section{References}

1 Henze, K. and Martin, W. (2003) Evolutionary biology: essence of mitochondria. Nature 426, 127-128

2 Pearce, S. et al. (2012) Mitochondrial diseases: translation matters. Mol. Cell. Neurosci. 55, 1-12

3 Schmitt, M.W. et al. (2012) Detection of ultra-rare mutations by nextgeneration sequencing. Proc. Natl. Acad. Sci. U.S.A. 109, 1450814513

4 Payne, B.A. et al. (2013) Universal heteroplasmy of human mitochondrial DNA. Hum. Mol. Genet. 22, 384-390

5 Ciccone, S. et al. (2013) Parkinson's disease: a complex interplay of mitochondrial DNA alterations and oxidative stress. Int. J. Mol. Sci. $14,2388-2409$

6 Smith, R.A. et al. (2012) Mitochondrial pharmacology. Trends Pharmacol. Sci. 33, 341-352

7 Karunadharma, P.P. et al. (2010) Mitochondrial DNA damage as a potential mechanism for age-related macular degeneration. Invest. Ophthalmol. Vis. Sci. 51, 5470-5479

8 Hauser, D.N. and Hastings, T.G. (2013) Mitochondrial dysfunction and oxidative stress in Parkinson's disease and monogenic parkinsonism. Neurobiol. Dis. 51, 35-42

9 Maresca, A. et al. (2013) The optic nerve: a "mito-window" on mitochondrial neurodegeneration. Mol. Cell. Neurosci. 55, 62-76

$10 \mathrm{Yu}$-Wai-Man, P. et al. (2011) Pattern of retinal ganglion cell loss in dominant optic atrophy due to OPA1 mutations. Eye 25, 596-602

11 Newman, N.J. (2012) Treatment of hereditary optic neuropathies. Nat. Rev. Neurol. 8, 545-556

12 Barot, M. et al. (2011) Mitochondrial dysfunction in retinal diseases. Curr. Eye Res. 36, 1069-1077

13 Duno, M. et al. (2013) A novel mitochondrial mutation m.8989G $>$ C associated with neuropathy, ataxia, retinitis pigmentosa: the NARP syndrome. Gene 515, 372-375

14 Comte, C. et al. (2013) Mitochondrial targeting of recombinant RNAs modulates the level of a heteroplasmic mutation in human mitochondrial DNA associated with Kearns Sayre Syndrome. Nucleic Acids Res. 41, 418-433

15 Koopman, W.J. et al. (2013) OXPHOS mutations and neurodegeneration. EMBO J. 32, 9-29

16 Itoh, K. et al. (2013) Mitochondrial dynamics in neurodegeneration. Trends Cell Biol. 23, 64-71

17 Kelly, R.D. et al. (2012) Mitochondrial DNA copy number is regulated in a tissue specific manner by DNA methylation of the nuclearencoded DNA polymerase gamma A. Nucleic Acids Res. 40, 1012410138

18 Lax, N.Z. et al. (2012) Sensory neuronopathy in patients harbouring recessive polymerase $\gamma$ mutations. Brain $135,62-71$

19 Nicholls, T.J. et al. (2013) Mitochondria: mitochondrial RNA metabolism and human disease. Int. J. Biochem. Cell Biol. 45, $845-849$

20 Mansergh, F.C. et al. (1999) Retinitis pigmentosa and progressive sensorineural hearing loss caused by a C12258A mutation in the mitochondrial MTTS2 gene. Am. J. Hum. Genet. 64, 971-985

21 Goto, Y. et al. (1990) A mutation in the tRNA(Leu)(UUR) gene associated with the MELAS subgroup of mitochondrial encephalomyopathies. Nature 348, 651-653
22 Cree, L.M. et al. (2008) A reduction of mitochondrial DNA molecules during embryogenesis explains the rapid segregation of genotypes. Nat. Genet. 40, 249-254

23 Tsao, K. et al. (1999) Smoking as an aetiological factor in a pedigree with Leber hereditary optic neuropathy. Br. J. Ophthalmol. 83, $577-581$

24 Carelli, V. et al. (2007) Grand rounds: could occupational exposure to n-hexane and other solvents precipitate visual failure in Leber hereditary optic neuropathy? Environ. Health Perspect. 115, 113-115

25 Mackey, D.A. et al. (2003) Leber's hereditary optic neuropathy triggered by antiretroviral therapy for human immunodeficiency virus. Eye 17, 312-317

26 Shankar, S.P. et al. (2008) Evidence for a novel x-linked modifier locus for Leber hereditary optic neuropathy. Ophthalmic Genet. 29, 17-24

27 Butler, E.K. et al. (2012) The mitochondrial chaperone protein TRAP1 mitigates $\alpha$-Synuclein toxicity. PLoS Genet. 8, e1002488

28 Marcuello, A. et al. (2009) Human mitochondrial variants influence on oxygen consumption. Mitochondrion 9, 27-30

29 Hudson, G. et al. (2007) Clinical expression of Leber hereditary optic neuropathy is affected by the mitochondrial DNA-haplogroup background. Am. J. Hum. Genet. 81, 228-233

30 Gómez-Durán, A. et al. (2012) Oxidative phosphorylation differences between mitochondrial DNA haplogroups modify the risk of Leber's hereditary optic neuropathy. Biochim. Biophys. Acta 1822, 12161222

31 Ridge, P.G. et al. (2012) Mitochondrial genomic analysis of late onset Alzheimer's disease reveals protective haplogroups H6A1A/ H6A1B: The Cache County Study on Memory in Aging. PLoS ONE 7, e45134

32 Mueller, E.E. et al. (2012) Mitochondrial haplogroups and control region polymorphisms in age-related macular degeneration: a casecontrol study. PLoS ONE 7, e30874

33 Gómez-Durán, A. et al. (2010) Unmasking the causes of multifactorial disorders: OXPHOS differences between mitochondrial haplogroups. Hum. Mol. Genet. 19, 3343-3353

34 Bratic, A. and Larsson, N.G. (2013) The role of mitochondria in aging. J. Clin. Invest. 123, 951-957

35 Trifunovic, A. et al. (2004) Premature ageing in mice expressing defective mitochondrial DNA polymerase. Nature 429, 417-423

36 Trifunovic, A. et al. (2005) Somatic mtDNA mutations cause aging phenotypes without affecting reactive oxygen species production. Proc. Natl. Acad. Sci. U.S.A. 102, 17993-17998

37 King, M.P. and Attardi, G. (1989) Human cells lacking mtDNA: repopulation with exogenous mitochondria by complementation. Science 246, 500-503

38 Ferreira, I.L. et al. (2011) Bioenergetic dysfunction in Huntington's disease human cybrids. Exp. Neurol. 231, 127-134

39 Arzuffi, P. et al. (2012) Partial tandem duplication of mtDNAtRNA(Phe) impairs mtDNA translation in late-onset mitochondrial myopathy. Neuromuscul. Disord. 22, 50-55

40 Desquiret-Dumas, V. et al. (2012) Metabolically induced heteroplasmy shifting and l-arginine treatment reduce the energetic defect in a neuronal-like model of MELAS. Biochim. Biophys. Acta 1822, 1019-1029

41 Abramov, A.Y. et al. (2010) Mechanism of neurodegeneration of neurons with mitochondrial DNA mutations. Brain 133, 797-807

42 Iyer, S. et al. (2012) Mitochondrial gene replacement in human pluripotent stem cell-derived neural progenitors. Gene Ther. 19, 469-475

43 Burbulla, L.F. et al. (2010) Dissecting the role of the mitochondrial chaperone mortalin in Parkinson's disease: functional impact of disease-related variants on mitochondrial homeostasis. Hum. Mol. Genet. 19, 4437-4452

44 Iyer, S. et al. (2012) Mitochondrial gene therapy improves respiration, biogenesis, and transcription in G11778A Leber's hereditary optic neuropathy and T8993G Leigh's syndrome cells. Hum. Gene Ther. 23, 647-657

45 Seibler, P. et al. (2011) Mitochondrial Parkin recruitment is impaired in neurons derived from mutant PINK1 iPS cells. J. Neurosci. 31, 5970-5976

46 Cooper, O. et al. (2012) Pharmacological rescue of mitochondrial deficits in iPSC-derived neural cells from patients with familial Parkinson's disease. Sci. Transl. Med. 4, 141ra90 
47 Tyynismaa, H. and Suomalainen, A. (2009) Mouse models of mitochondrial DNA defects and their relevance for human disease. EMBO Rep. 10, 137-143

48 Quintana, A. et al. (2010) Complex I deficiency due to loss of Ndufs4 in the brain results in progressive encephalopathy resembling Leigh syndrome. Proc. Natl. Acad. Sci. U.S.A. 107, 10996-11001

49 Dunn, D.A. et al. (2012) Animal models of human mitochondrial DNA mutations. Biochim. Biophys. Acta 1820, 601-607

50 Kujoth, G.C. et al. (2005) Mitochondrial DNA mutations, oxidative stress, and apoptosis in mammalian aging. Science 309, 481-484

51 Milenkovic, D. et al. (2013) TWINKLE is an essential mitochondrial helicase required for synthesis of nascent D-loop strands and complete mtDNA replication. Hum. Mol. Genet. 22, 1983-1993

52 Dunn, D.A. and Pinkert, C.A. (2012) Nuclear expression of a mitochondrial DNA gene: mitochondrial targeting of allotopically expressed mutant ATP6 in transgenic mice. J. Biomed. Biotechnol. http://dx.doi.org/10.1155/2012/541245

53 Ellouze, S. et al. (2008) Optimized allotopic expression of the human mitochondrial ND4 prevents blindness in a rat model of mitochondrial dysfunction. Am. J. Hum. Genet. 83, 373-387

54 Qi, X. et al. (2007) The mutant human ND4 subunit of complex I induces optic neuropathy in the mouse. Invest. Ophthalmol. Vis. Sci. $48,1-10$

55 Cannon, M.V. et al. (2011) Xenomitochondrial mice: investigation into mitochondrial compensatory mechanisms. Mitochondrion 11, 33-39

56 Nakada, K. and Hayashi, J. (2011) Transmitochondrial mice as models for mitochondrial DNA-based diseases. Exp. Anim. 60, $421-431$

57 Lin, C.S. et al. (2012) Mouse mtDNA mutant model of Leber hereditary optic neuropathy. Proc. Natl. Acad. Sci. U.S.A. 109, 20065-20070

58 Shokolenko, I.N. et al. (2010) The approaches for manipulating mitochondrial proteome. Environ. Mol. Mutagen. 51, 451-461

59 Heller, A. et al. (2012) Targeting drugs to mitochondria. Eur. J. Pharm. Biopharm. 82, 1-18

60 Mileshina, D. et al. (2011) Mitochondrial transfection for studying organellar DNA repair, genome maintenance and aging. Mech. Ageing Dev. 132, 412-423

61 Niazi, A.K. et al. (2012) Targeting nucleic acids into mitochondria: progress and prospects. Mitochondrion http://dx.doi.org/10.1016/ j.mito.2012.05.004

62 Yasuzaki, Y. et al. (2010) Mitochondrial matrix delivery using MITOPorter, a liposome-based carrier that specifies fusion with mitochondrial membranes. Biochem. Biophys. Res. Commun. 397, $181-186$

63 Yamada, Y. and Harashima, H. (2012) Enhancement in selective mitochondrial association by direct modification of a mitochondrial targeting signal peptide on a liposomal based nanocarrier. Mitochondrion http://dx.doi.org/10.1016/j.mito.2012.09.001

64 Lyrawati, D. et al. (2011) Expression of GFP in the mitochondrial compartment using DQAsome-mediated delivery of an artificial minimitochondrial genome. Pharm. Res. 28, 2848-2862

65 Smith, R.A. and Murphy, M.P. (2011) Mitochondria-targeted antioxidants as therapies. Discov. Med. 11, 106-114

66 Smith, R.A. and Murphy, M.P. (2010) Animal and human studies with the mitochondria-targeted antioxidant MitoQ. Ann. N. Y. Acad. Sci. 1201, 96-103

67 Szeto, H.H. and Schiller, P.W. (2011) Novel therapies targeting inner mitochondrial membrane: from discovery to clinical development. Pharm. Res. 28, 2669-2679

68 Keeney, P.M. et al. (2009) Mitochondrial gene therapy augments mitochondrial physiology in a Parkinson's disease cell model. Hum. Gene Ther. 20, 897-907

69 Jash, S. et al. (2012) Modulation of mitochondrial respiratory capacity by carrier-mediated transfer of RNA in vivo. Mitochondrion 12, 262-270

70 Wang, Y. et al. (2013) Systemic delivery of modified mRNA encoding herpes simplex virus 1 thymidine kinase for targeted cancer gene therapy. Mol. Ther. 21, 358-367

71 Mercer, T.R. et al. (2011) The human mitochondrial transcriptome. Cell 146, 645-658

72 Wang, G. et al. (2012) Correcting human mitochondrial mutations with targeted RNA import. Proc. Natl. Acad. Sci. U.S.A. 109, 4840-4845
73 Zimmer, K.P. et al. (1999) Efficient mitochondrial import of newly synthesized ornithine transcarbamylase (OTC) and correction of secondary metabolic alterations in spf(ash) mice following gene therapy of OTC deficiency. Mol. Med. 5, 244-253

74 Wang, L. et al. (2012) Sustained correction of OTC deficiency in spf(ash) mice using optimized self-complementary AAV2/8 vectors. Gene Ther. 19, 404-410

$75 \mathrm{Di}$ and Meo, I. et al. (2012) Effective AAV-mediated gene therapy in a mouse model of ethylmalonic encephalopathy. EMBO Mol. Med. 4, 1008-1014

$76 \mathrm{Yu}, \mathrm{H}$. et al. (2012) Gene delivery to mitochondria by targeting modified adenoassociated virus suppresses Leber's hereditary optic neuropathy in a mouse model. Proc. Natl. Acad. Sci. U.S.A. 109, E1238-E1247

77 Marella, M. et al. (2010) Successful amelioration of mitochondrial optic neuropathy using the yeast NDI1 gene in a rat animal model. PLoS ONE 5, e11472

78 Chadderton, N. et al. (2013) Intravitreal delivery of AAV-NDI1 provides functional benefit in a murine model of Leber hereditary optic neuropathy. Eur. J. Hum. Genet. 21, 62-68

79 Bacman, S.R. et al. (2011) Manipulation of mtDNA heteroplasmy in all striated muscles of newborn mice by AAV9-mediated delivery of a mitochondria-targeted restriction endonuclease. Gene Ther. http:// dx.doi.org/10.1038/gt.2011.196

80 Mancuso, M. et al. (2012) Drugs and mitochondrial diseases: 40 queries and answers. Expert Opin. Pharmacother. 13, 527-543

81 Sadun, A.A. et al. (2012) Effect of EPI-743 on the clinical course of the mitochondrial disease Leber hereditary optic neuropathy. Arch. Neurol. 69, 331-338

82 Klopstock, T. et al. (2011) A randomized placebo-controlled trial of idebenone in Leber's hereditary optic neuropathy. Brain 134, $2677-$ 2686

83 Tachibana, M. et al. (2013) Towards germline gene therapy of inherited mitochondrial diseases. Nature 493, 627-631

84 Paull, D. et al. (2013) Nuclear genome transfer in human oocytes eliminates mitochondrial DNA variants. Nature 493, 632-637

85 Tachibana, M. et al. (2009) Mitochondrial gene replacement in primate offspring and embryonic stem cells. Nature 461, 367-372

86 Craven, L. et al. (2010) Pronuclear transfer in human embryos to prevent transmission of mitochondrial DNA disease. Nature 465, 82-85

87 Giordano, C. et al. (2011) Oestrogens ameliorate mitochondrial dysfunction in Leber's hereditary optic neuropathy. Brain 134, $220-234$

88 Zhang, X. et al. (2002) Mouse model of optic neuropathy caused by mitochondrial complex I dysfunction. Neurosci. Lett. 326, 97-100

89 Przedborski, S. et al. (2004) MPTP as a mitochondrial neurotoxic model of Parkinson's disease. J. Bioenerg. Biomembr. 36, 375-379

90 Kruse, S.E. et al. (2008) Mice with mitochondrial complex I deficiency develop a fatal encephalomyopathy. Cell Metab. 7, 312-320

91 Gispert, S. et al. (2009) Parkinson phenotype in aged PINK1-deficient mice is accompanied by progressive mitochondrial dysfunction in absence of neurodegeneration. PLoS ONE 4, e5777

92 Klein, J.A. et al. (2002) The harlequin mouse mutation downregulates apoptosis-inducing factor. Nature 419, 367-374

93 Bénit, P. et al. (2008) The variability of the harlequin mouse phenotype resembles that of human mitochondrial-complex Ideficiency syndromes. PLoS ONE 3, e3208

94 Graziewicz, M.A. et al. (2004) Structure-function defects of human mitochondrial DNA polymerase in autosomal dominant progressive external ophthalmoplegia. Nat. Struct. Mol. Biol. 11, 770-776

95 Han, C. and Someya, S. (2013) Mouse models of age-related mitochondrial neurosensory hearing loss. Mol. Cell. Neurosci. 55, $95-100$

96 Spelbrink, J.N. et al. (2001) Human mitochondrial DNA deletions associated with mutations in the gene encoding Twinkle, a phage T7 gene 4-like protein localized in mitochondria. Nat. Genet. 28, 223-231

97 Tyynismaa, H. et al. (2005) Mutant mitochondrial helicase Twinkle causes multiple mtDNA deletions and a late-onset mitochondrial disease in mice. Proc. Natl. Acad. Sci. U.S.A. 102, 17687-17692

98 Graham, B.H. et al. (1997) A mouse model for mitochondrial myopathy and cardiomyopathy resulting from a deficiency in the heart/muscle isoform of the adenine nucleotide translocator. Nat. Genet. 16, 226-234 
99 Fan, W. et al. (2008) A mouse model of mitochondrial disease reveals germline selection against severe mtDNA mutations. Science 319 , 958-962

100 Inoue, K. et al. (2000) Generation of mice with mitochondrial dysfunction by introducing mouse mtDNA carrying a deletion into zygotes. Nat. Genet. 26, 176-181

101 Nakada, K. et al. (2004) Accumulation of pathogenic DeltamtDNA induced deafness but not diabetic phenotypes in mito-mice. Biochem. Biophys. Res. Commun. 323, 175-184

102 Yokota, M. et al. (2010) Generation of trans-mitochondrial mito-mice by the introduction of a pathogenic G13997A mtDNA from highly metastatic lung carcinoma cells. FEBS Lett. 584, 3943-3948

103 Raper, S.E. et al. (2002) A pilot study of in vivo liver-directed gene transfer with an adenoviral vector in partial ornithine transcarbamylase deficiency. Hum. Gene Ther. 13, 163-175

104 Jarraya, B. et al. (2009) Dopamine gene therapy for Parkinson's disease in a nonhuman primate without associated dyskinesia. Sci. Transl. Med. 1, 2ra4
105 Quintana, A. et al. (2012) Fatal breathing dysfunction in a mouse model of Leigh syndrome. J. Clin. Invest. 122, 2359-2368

106 Bouaita, A. et al. (2012) Downregulation of apoptosis-inducing factor in Harlequin mice induces progressive and severe optic atrophy which is durably prevented by AAV2-AIF1 gene therapy. Brain $135,35-52$

107 Barber-Singh, J. et al. (2010) Protective role of rAAV-NDI1, serotype 5, in an acute MPTP mouse Parkinson's model. Parkinsons Dis. 2011, 438370

108 Seo, B.B. et al. (2006) In vivo complementation of complex I by the yeast Ndi1 enzyme. Possible application for treatment of Parkinson disease. J. Biol. Chem. 281, 14250-14255

109 Marella, M. et al. (2008) Protection by the NDI1 gene against neurodegeneration in a rotenone rat model of Parkinson's disease. PLoS ONE 3, e1433

110 Morais, V.A. et al. (2009) Parkinson's disease mutations in PINK1 result in decreased Complex I activity and deficient synaptic function. EMBO Mol. Med. 1, 99-111 